\title{
Énonciation et modélisation du réel dans Contours du jour qui vient de Léonora Miano
}

\author{
Ladislas Nzessé \\ Université de Dschang et École normale supérieure de \\ Yaoundé (Cameroun)
}

Tout énoncé, avant d'être ce fragment de langue naturelle que la linguistique s'efforce d'analyser, est le produit d'un événement unique, son énonciation, qui suppose un énonciateur, un destinataire, un moment et un lieu particulier. Cet ensemble d'éléments définit la situation d'énonciation.

Maingueneau, 1991, p. 6

Léonora Miano a choisi pour son roman un titre pour le moins ambitieux. Il y a chez cette écrivaine une volonté de 
représentation affichée dès le titre : Contours du jour qui vient. L'association de deux substantifs, dont l'un (le contour) s'associe aux éléments propres à une représentation précise et l'autre (le jour) peut échapper à toute représentation précise, crée dans l'esprit du lecteur quelque chose d'inattendu, d'autant plus que l'évocation «du jour qui vient» comporte une imprécision établie par l'article défini « du». Le titre, tel qu'il apparaît, annonce une certaine manière de dire et de représenter. Il y a là une volonté non seulement de créer, mais de déboucher sur une note d'espoir. C'est du moins le sens de la phrase " The winds of change are blowing », mise en exergue en début de roman. En décidant d'envisager un temps futur, la romancière s'oppose du même coup au temps présent, forcément défectueux. S'il est vrai que la représentation a à voir avec le réel, il est intéressant de voir comment le roman rend compte d'une nouvelle manière d'envisager le réel et, surtout, d'examiner les procédés mis en place par l'auteure. Il ne fait aucun doute qu'il y a dans cette œuvre une possible corrélation entre la création littéraire et l'environnement socioculturel. Et comme le pense à juste titre Vladimir Nabokov, «l'art d'écrire est un art très futile s'il n'implique pas avant tout l'art de voir le monde comme un potentiel » (1985, p.40-41). Notre projet consiste ici à montrer que c'est dans et par le discours ${ }^{1}$ que le sujet parlant (communiquant) construit, consciemment ou inconsciemment, volontairement ou involontairement, sa propre identité et l'identité collective (de l'Afrique). Nous

\footnotetext{
1 Nous considérons ici le discours au sens de Guespin. C'est ce qui s'oppose à l'énoncé, c'est-à-dire que "l'énoncé, c'est la suite des phrases émises entre deux blancs sémantiques, deux arrêts de la communication; le discours, c'est l'énoncé considéré du point de vue du mécanisme discursif qui le conditionne ». (1971, p. 10)
} 
empruntons à l'analyse du discours ${ }^{2}$ et à l'ethnostylistique ${ }^{3}$ les outils méthodologiques pour cette étude. Ceci étant, nous étudierons, d'une part, la narration à la première personne et, d'autre part, l'usage de l'onomastique des personnages et des lieux.

\section{La narration à la première personne}

Dans Contours $d u$ jour qui vient, la narration semble assumée par un « je » qui interpelle constamment un «tu» :

Il n'est plus que des ombres alentour, je suis l'une d'elles et c'est à toi que je pense. La dernière fois que nous nous sommes vues, tu m'avais attachée sur mon lit. Tu m'avais rossée de toutes tes forces avant de convoquer nos voisins, afin qu'ils voient ce que tu comptais faire de cet esprit malin qui vivait sous ton toit et se disait ta fille. » $(C J V$, p. 15)

\footnotetext{
2 Grawitz soutient que toutes les recherches en ce domaine partent « [...] du principe que les énoncés ne se présentent pas comme des phrases ou des suites de phrases mais comme des textes. Or un texte est un mode d'organisation spécifique qu'il faut étudier comme tel en le rapportant aux conditions dans lesquelles il est produit. Considérer la structure d'un texte en le rapportant à ses conditions de production, c'est l'envisager comme discours. » (1990, p. 345)

3 Réfléchissant sur les particularités esthétiques du texte négro-africain, Mendo Zé élabore le concept d'ethnostylistique, qu'il définit comme «une stylistique qui a pour objet la critique du style des textes littéraires, pour procédé les techniques d'analyse en science du langage, et pour finalité la prise en compte des conditions de production et de réception des textes ainsi que l'étude des modes particuliers de leurs valeurs culturelles » (2004, p. 1920). S'agissant particulièrement du texte africain francophone et de ses particularités formelles, l'ethnostylistique présuppose son articulation à un cadre de référence ethnologique où l'œuvre littéraire, "se définissant par sa spécificité esthétique, révèle son appartenance à une matrice textuelle que l'on peut définir comme un architexte » (Noumssi, 2004, p. 82). Cet architexte peut se ramener à un corpus dont les traits (linguistiques géographiques et historiques) caractérisent une manière de narration, suivant l'aire ethnoculturelle.
} 
L'instance narrative s'identifie à la première personne du singulier et s'inscrit du même coup dans un ancrage subjectif. C'est la matérialisation de la présence effective d'un énonciateur à travers ce que Benveniste appelle «l'accentuation de la relation discursive au partenaire, que celui-ci soit réel ou imaginaire, individuel ou collectif » (1966, p. 85). Dans le même ordre d'idées, il affirme que «le langage est ainsi organisé tel qu'il permet à chaque locuteur de s'approprier la langue entière en se désignant comme "je". Les pronoms personnels sont le premier point d'appui pour cette mise au jour de la subjectivité dans le langage. » (1966, p. 262)

Le lecteur de Léonora Miano est très tôt placé en face d'une énonciation discursive avec tous les éléments de la déixis requis : les personnes de l'énonciation (je, tu, nous), les temps de l'énonciation (présent, imparfait, plus-que-parfait), le cadre spatio-temporel qui suppose un « ici » et un «maintenant». Le roman emprunte largement au style de la conversation courante, ce qui ne peut qu'accentuer l'illusion de vraisemblance. La conversation a l'avantage d'être informationnelle pour le lecteur, car si ce dernier ne peut encore à ce stade du roman répondre avec précision aux questions qui? quoi? où?, il sait déjà précisément qu'il est question d'un rapport conflictuel entre une mère et sa fille. En instaurant d'ailleurs un tel rapport conflictuel, la narratrice crée, en posant les deux personnages comme vrais, une illusion de réalité dans l'esprit du lecteur.

L'adresse à la mère lancée dès le début du roman permet l'irruption dans la diégèse d'un «tu » dont on n'apprendra que bien plus tard qu'il s'agit d'Ewenji. L'usage de la première personne dans la narration romanesque participe d'un artifice 
dont le mérite est à la fois de créer plus facilement un univers dans un cadre spatio-temporel qui semble assez proche, de même qu'il prend le lecteur à témoin. C'est aussi ce que semblent traduire ces propos de René Demoris :

La première personne a du moins l'avantage de renvoyer à un sujet chez qui cette pensée, quelle que soit sa pertinence, a été réalité. [...] C'est donc au moment où il se définit contre la logique "naturelle » du récit que l'être éprouve le mieux sa propre vérité. Le récit le plus vrai reste cependant le récit personnel puisqu'il intègre l'illusion d'avoir une histoire. (2002, p. 337)

David Ndachi Tagne trouve quant à lui une autre explication à l'usage de la première personne dans les romans d'Afrique subsahariens. Cela tiendrait, d'après lui, à une certaine corrélation entre la création et l'environnement social :

Quoi qu'en disent les formalistes, la corrélation entre la création littéraire et l'environnement social est une donnée qu'il faut examiner en permanence sous le ciel africain. [...] Le roman à la première personne apparaîtra en effet pour nombre d'auteurs comme le tremplin d'expression de leurs expériences intimes. (1996, p. 109)

Le degré d'implication du narrateur dans la diégèse en fait un acteur principal. Le lecteur ne peut négliger la présence de celui qui raconte l'histoire quand ce dernier s'exprime à la première personne. On a envie de suivre cette voix qui se donne comme une conscience, à laquelle le lecteur est tenté de s'identifier. La narration change de ton lorsque le « je » de départ se mue en «nous». C'est du moins ce qui se passe assez souvent dans Contours du temps qui vient, lorsque la narratrice prend en charge tout le destin d'un peuple:

Nous sommes ce peuple d'oralité qui ne dit jamais rien d'essentiel, qui ne sait faire que des bruits pour tenter 
d'étouffer la douleur. Nous sommes des adorateurs de la parole futile ou prosaïque. Taire l'intime nous demande tant d'efforts qu'il n'est pas surprenant que nous soyons fous et exsangues. La plupart d'entre nous. Pas nous tous. Des voix refuseront de se taire. (CJV, p. 195-196)

En affirmant ainsi son identité culturelle, Musango rend possible l'identification avec le lecteur africain. La culture de l'oralité n'est-elle pas caractéristique des peuples africains? C'est une narratrice à visage humain qui se présente ainsi au lecteur, avec un sens du patriotisme qui ne peut qu'interpeller. Par ailleurs, l'usage de l'épanorthose (" pas nous »), ne peut que légitimer un discours déjà hautement subjectif. La narratrice semble en savoir beaucoup plus sur l'histoire qu'elle raconte.

De plus, l'alternance entre le « je » et le « nous » témoigne de la volonté de la narratrice de prendre en charge un discours individuel et collectif, de même qu'elle rend compte des différents parcours. C'est à juste titre qu'Odile Cazenave déclare, dans son ouvrage consacré à une étude sur les nouveaux romanciers issus de l'immigration :

L'alternance entre le «je » et le «nous » signale aussi le lien étroit entre le discours individuel et le discours collectif, entre expérience individuelle et collective. Ce parcours va de pair également avec un parcours géographique : du village à la ville, du Cameroun à Paris, de l'enfance à l'adolescence, à l'âge adulte, de la vie de célibataire à celle de femme mariée. (2003, p. 106)

La forme romanesque ainsi présentée devient le lieu de la recherche d'une nouvelle éthique littéraire et culturelle, car les romanciers de la nouvelle génération, contrairement à leurs prédécesseurs, vivent pour la plupart en France. Ils offrent un regard de nature et de portées différentes. Le regard n'est plus nécessairement tourné vers l'Afrique, mais plutôt sur soi. Cette dynamique nouvelle va créer ce que Cazenave appelle un 
" décentrage de l'écriture ». Le travail de l'écriture se fait par et à travers le choix de la forme narrative. Et Léonora Miano a de bonnes raisons d'opter pour la forme personnelle.

L'usage d'un « je » narrant participe dans ce roman d'une volonté à la fois de réalisme et de sacralisation du récit. L'impression de réalité est renforcée par l'emploi du présent, comme on peut le voir dans ce passage :

La douleur est si vive qu'on a le sentiment que les pieds pleurent. L'asphalte succède à la poussière. Sur l'autre, je sens les restes de la chaleur du jour. Ici, le froid ne descend pas au dessous de quinze degrés. Il y a des détritus sur le sol. Des tessons de bouteilles, des morceaux de fil de fer, des échardes roublardes cachées là où l'œil humain ne peut les voir. Je marche en regardant où je pose les pieds. Les échardes mes piquent tout de même. Elles s'enfoncent dans ma chair. Je n'essaie pas de les enlever. (CJV, p. 120)

Au-delà de la description d'une misère quotidienne, ce passage a ceci de particulier qu'il plonge le lecteur au cœur de l'action. Par l'utilisation du présent, le discours se donne comme vrai, et le lecteur natif ne s'y perdrait pas, car on est bien devant une scène de vie quotidienne. Ce passage a aussi l'avantage d'associer au présent la force du déictique «ici» et du présentatif existentiel « il y a ». La narratrice ne manque pas de donner au lecteur averti des indices qui lui permettent de reconstituer des réalités spatio-temporelles à partir des données textuelles. Le reflet du réel peut ainsi se dégager de la création fictionnelle. En effet, comment ne pas établir un lien entre le «Mboasu » et le "Cameroun » quand on sait que c'est par ce nom que le peuple Sawa du Cameroun désigne, en langue duala, le village, la terre natale ou le pays d'origine? 
Plus loin, lorsque Musango parle de la venue de la Française Aïda, elle rappelle à la mémoire collective un fait historique majeur : «À l'époque où elle [la Française Aïda] était venue vivre au Mboasu, la situation économique était similaire à celle de la Corée du Sud.» (CJV, p. 32) Cette information capitale ne saurait être le fait d'un hasard de l'écriture, car elle sert de passerelle entre le lecteur et l'auteur. L'information se donne ainsi comme un point de repère historique. Il y a là un héritage culturel à partager. Tout Camerounais qui n'a rien perdu de sa mémoire peut se rappeler l'époque où on aimait à établir la comparaison avec la Corée du Sud. C'est là une allusion et une référence à l'actualité événementielle du Cameroun. Tout se passe comme si le texte de Miano était une espèce de puzzle dont le lecteur aurait la charge de reconstituer les parties. Ce procédé est le produit de la narration homodiégétique, qui permet l'irruption du discours dans la diégèse comme un flux de la conscience, car comme le souligne Genette, «[1]a narration homodiégétique par nature ou convention (en l'occurrence c'est tout un), simule l'autobiographie bien plus étroitement que la narration hétérodiégétique ne simule ordinairement le récit historique » (1983, p. 52). Le lecteur peut se rendre compte que, dans le texte, se trouve disséminé un nombre d'informations se rapportant directement à la réalité socioculturelle du Cameroun. C'est ainsi que lorsqu'on lit une phrase aussi révélatrice que celle-ci : "Les habitants du Mboasu, qui ont pourtant une souche linguistique commune, ne se comprennent pas d'une région à l'autre, d'un bout de terrain à l'autre. » (CJV, p. 203), on n'a aucun mal à faire le lien une fois de plus entre le Mboasu (pays imaginaire) et le Cameroun pays réel. Les études de linguistique africaine ont permis de mettre au jour une 
réalité linguistique certaine: le pays compte à lui tout seul environ deux cent quatre-vingts unités-langues répertoriées. Affirmer que, d'un bout de terrain à l'autre, les habitants de ce pays ne se comprennent pas ne relève donc pas du tout de la fiction.

En outre, la première personne de narration se saisit dans une dynamique de communication propre au roman africain. Elle suppose pour bon nombre de romanciers africains une large part d'investissement personnel. C'est ce que semble expliquer l'écrivaine Calixthe Beyala :

J'utilise le «je» car j'habite mes personnages. Je les habite physiquement, je suis à leur place au moment précis où j'écris. Je suis avec eux dans leur environnement. Je n'utilise pas la troisième personne parce que «il» ou «elle» suppose une distance et je n'éprouve pas cette distance lorsque j'écris. (2003, p. 44)

Cette romancière exprime là à sa manière l'étroitesse des rapports pouvant exister entre un auteur et ses textes. Le même constat pourrait se faire chez Miano, dont le texte semble balisé de nombreuses allusions et de références à une actualité culturelle ou événementielle du pays d'origine. Ainsi, la ville imaginaire de Sombé, de même que son fleuve la Tubé, cités dans ses deux romans, font étrangement penser à la ville de Douala et à son fleuve, le Wouri. Le village des pêcheurs, quant à lui, ne manque pas d'évoquer dans l'imaginaire du lecteur natif le célèbre village des pêcheurs «Youpwé », situé dans une des banlieues de la ville. Ce n'est pas en vain que la romancière donne cette information capitale :

Il ne fallait pas oublier que c'était par l'estuaire de la Tubé qu'arrivaient jadis les explorateurs et les commerçants européens, et que Sombé avait été leur unique comptoir au Mboasu pendant des décennies! Les gens du Sud ne craignaient 
plus assez les Blancs qui les avaient pris en grippe, et avaient confié le pouvoir à ceux qui militaient pour le Mboasu français. » (CJV, p. 57)

Cette citation est à mettre en relation avec les nombreuses autres informations qui ont toutes un point commun : l'histoire du Cameroun. Ne faut-il pas y voir un besoin de sauvegarde de la mémoire commune? Ces informations livrées de manière éparse seraient alors des mémorandums pour que jamais personne n'oublie que le Cameroun doit son nom au fleuve Wouri, situé à Douala, fleuve dans lequel des explorateurs portugais découvrirent des crevettes en grand nombre et décidèrent d'appeler le lieu "rio dos camaroes ", rivière des crevettes. Pour que personne n'oublie aussi qu'avant, bien avant l'indépendance, le Cameroun fut mis sous la tutelle de deux grandes puissances européennes : la France et la GrandeBretagne. Il existait alors un Cameroun français et un Cameroun anglais. Le pays tient d'ailleurs ses deux langues officielles (le français et l'anglais) de l'intervention de ces puissances dans son histoire.

Le roman se ménage ainsi de nombreux points d'histoire, pour que jamais personne n'oublie. Il convient de noter cependant que ces points d'histoire sont à décoder par le lecteur natif; pour les autres lecteurs, ils peuvent n'avoir aucun sens. Ici se pose avec acuité la délicate question de la réception des textes africains, de même que le problème de la langue et de l'identité culturelle. Les écrivains africains francophones se trouvent pris dans une interrelation culturelle et identitaire du fait de leur appartenance à une double culture : africaine et francophone. C'est aussi ce qu'affirme Cazenave lorsqu'elle déclare : 
La question de langue et identité est centrale à la littérature francophone en général et au roman africain en particulier. Il s'agit en effet pour l'écrivain d'exprimer à travers une langue autre, le français, des aspects culturels, des concepts philosophiques ou religieux, qui n'existent pas nécessairement dans la culture et la langue française. La tension linguistique qui en résulte et la présence d'un double lectorat, africain et français, contribuent à l'inscription dans le roman [...] du dilemme identitaire des personnages face à un mode de vie et de pensée autres. Cette interrelation entre langue et identité passe au premier plan dans les nouvelles écritures de soi. (2003, p. 49)

Dès lors, Contours $d u$ jour qui vient, $\mathrm{du}$ fait de l'homodiégétisation du récit et de la place du présent de l'indicatif, se situe dans cette nouvelle dynamique d'écriture des avant-gardes. Si le roman a besoin de l'écriture à la première personne pour créer l'illusion de réalité, cet artifice est rendu possible par l'usage du présent, qui permet la narration d'événements proches du lecteur. L'effet conjoint du narrateur homodiégétique et du présent contribue à rapprocher narrateur et lecteur à travers la valeur de simultanéité, comme le montre ce passage :

Je ne peux me résoudre à envisager la crédulité de l'assistance, de tous ces gens qui sont là. Il me semble au contraire que nul n'est dupe. Tout le monde sait qu'il y a une combine, et chacun espère découvrir le mot de passe pour faire partie du gang des millionnaires oints le dimanche. » (CJV, p. 198)

L'impression de réel se lit dans ce passage à travers la mise en discours du récit. Le discours de la narratrice apparaît comme le produit d'une conscience subjective. Cette subjectivité se trouve elle-même renforcée par l'usage de la négation («ne »), de l'épanorthose ( «au contraire »), et du déterminant indéfini à valeur hyperbolique («tout»). On conviendra avec Genette 
« que l'effet d'homodiégétisation n'est jamais totalement évacué d'un récit au présent dont le temps porte toujours plus ou moins présence d'un narrateur qui - pense inévitablement le lecteur - ne peut être bien loin d'une action qu'il donne luimême si proche » (1983, p. 55).

Il ne fait aucun doute que le lecteur ne peut que s'accommoder de cette forme d'écriture qui tend à le rapprocher du narrateur et de son histoire. Il arrive même qu'il soit pris de court lorsque le roman va puiser aux sources de la mémoire, comme le montrent ces propos de Musango : «À présent, je ne sais plus. Je me dis seulement que puisque je suis là, puisque tout est arrangé... Grand-mère revient avec le foyer. Mbalè se précipite pour l'aider, non sans m'avoir lancé un regard noir. » (CJV, p. 257) Avec cette interruption inattendue $\mathrm{du}$ récit qui confère définitivement au roman un aspect subjectif, on se croirait dans la rédaction de mémoires. Dire « je ne sais plus », c'est à la fois poser l'existence d'une conscience au monde et reconnaître les zones d'ombre de cette même conscience qui ne peut tout saisir. On est également très proche du monologue intérieur.

Il y a là un renouvellement de l'écriture du roman qui ne fait qu'emboîter le pas aux écrivains du Nouveau Roman et qui doit beaucoup aux littératures migrantes, lesquelles, du fait de leur appartenance à une double culture, posent un problème à l'historiographie littéraire. C'est aussi ce que semble dire quand elle affirme que :

Les littératures migrantes ont désormais acquis dans l'institution littéraire une visibilité qui accompagne le mouvement social de l'immigration et érode par là-même toutes les certitudes qui fondaient les notions d'identité et de culture nationale définies comme un ensemble de 
représentations d'institutions et d'affiliations fondées sur une conception homogène de la nation. L'émergence de ces écritures nouvelles aux marges des littératures nationales ont en effet créé une faille dans ces certitudes sécurisantes en faisant entendre d'autres voix, d'autres discours, d'autres positions à l'intérieur du même champ littéraire. (Albert, 1999, p. 85)

Il est donc important de prendre en compte la prolifération des cultures en Afrique comme une richesse pour la littérature de langue française. Chaque écriture particulière charrie une multitude d'expériences qui ont jalonné le parcours de l'écrivain en tant qu'être oscillant entre deux mondes, deux cultures, deux espaces. C'est l'écriture de l'entre-deux, dont la narration à la première personne semble la plus à même de rendre compte de tous les contours. La première personne de narration joue un rôle indéniable dans la crédibilisation du discours romanesque, la narratrice ne ménageant aucun effort pour solliciter l'adhésion du lecteur. Cette stratégie est encore remarquable dans l'onomastique des personnages et des lieux.

\section{L'onomastique des personnages et des lieux}

L'onomastique vise à tirer tous les renseignements possibles des noms propres, noms de lieu (toponymie) ou de personnes (anthroponymie). Selon Peremans,

[1]'onomastique étudie l'expansion des noms de personne et de lieu dans le temps et l'espace. Elle se distingue donc nettement de la prosopographie, dont le but est l'identification des personnages. Prenons un exemple: dans les papyrus, on rencontre souvent le nom Artémidore; l'onomastique essayera de préciser dans quelles régions d'Égypte ce nom a été employé, à quelle époque, dans quelle classe sociale, tandis 
qu'une étude prosopographique s'efforcera d'identifier les personnes qui ont porté ce nom. (1981, p. 49)

Le roman de Léonora Miano fait une part importante à l'onomastique, à la fois des personnages et des lieux. En effet, le choix des noms y est loin d'être anodin. On peut y voir un artifice lié à l'effet de réel créé par l'illusion romanesque; mais bien plus, ce pourrait être une manière pour la romancière de garder le contact avec le réel, car si on en croit Marthe Robert, «[l]a fortune historique du roman tient évidemment aux privilèges exorbitants que la littérature et la réalité lui ont concédés toutes deux avec la même générosité » (2004, p. 15). C'est là une manière de reconnaître le lien indissociable qui existe entre littérature et réalité. C'est dire aussi que si la littérature peut rendre compte d'une certaine réalité, la réalité, elle, a besoin, pour exister, d'être nommée. Vue sous cet angle, la littérature pourrait véhiculer la culture et c'est tout l'intérêt du choix du nom dans Contours du jour qui vient.

Les choix de la romancière ne manquent pas d'interpeller le lecteur et, plus particulièrement, le lecteur natif, à qui les noms rappellent un univers culturel assez particulier. S'il est vrai que le roman reste et demeure une œuvre de fiction et que les personnages et les événements narrés sont loin d'appartenir à un individu, force est de constater néanmoins que l'œuvre donne à voir une certaine réalité culturelle. Ainsi, le lecteur n'a aucun mal à identifier l'espace africain. Deux cas de figure vont se présenter : d'un côté, le lecteur non natif, qui saura identifier l'espace africain et, de l'autre, le lecteur natif, pour qui les noms de personnages et de lieux seront beaucoup plus révélateurs.

Le lecteur non africain qui s'engage dans la lecture de l'œuvre de Miano ne tarde pas à situer le Mboasu en Afrique 
équatoriale. En effet, nombreux sont les passages qui portent les indices de ce cadre spatial. Dès les premières pages, on peut lire :

On les [des évangélistes américains] voyait souvent rougir douloureusement au soleil, vêtus de chemises blanches à manches courtes et de pantalons noirs, et on se disait qu'ils avaient de bonnes raisons pour venir si loin de chez eux, souffrir sous l'ardent soleil de notre Afrique équatoriale. $(\mathrm{CJV}$, p. 25)

L'histoire a donc lieu en Afrique équatoriale et la narratrice s'identifie aux Africains à travers le déterminant possessif «notre». Plus loin, c'est une tournure périphrastique qui viendra mettre le lecteur sur la piste de l'Afrique : "Telle est cette terre première, le fameux berceau de l'humanité : elle n'engendre plus que des faits divers. » (CJV, p. 109) Le continent africain est souvent désigné, à tort ou à raison, comme le berceau de l'humanité. Cette périphrase, reprise à son compte par la romancière, ne manque pas d'évoquer un référent précis chez le lecteur, tout comme les détails relatifs à la végétation africaine. Le lecteur averti n'a en fait aucun mal à rattacher des végétaux tels que le manguier, le baobab, le papayer, le bambou ou l'hévéa à l'univers spatial africain. Cette terre d'Afrique équatoriale semble obséder la romancière qui, depuis L'Intérieur de la nuit, ne manque pas de multiplier les allusions allant dans ce sens.

$\mathrm{Au}$ fur et à mesure de la lecture, la situation géographique du Mboasu se fait de plus en plus précise. Des détails tels que «l'Afrique Équatoriale Française », « les peaux des Africains du centre » ne sauraient laisser le lecteur indifférent. On apprend par ces détails que le Mboasu se situe quelque part en Afrique centrale. Le détail le plus précis sera sans doute celui qui fait du 
Tchad un voisin immédiat du Mboasu, comme on peut le lire à travers ces lignes : « Des passeurs les emmènent par les déserts du Tchad et du Niger, vers la Méditérranée où des cannots les attendent. » (CJV, p. 51)

Le Tchad est, avec la Guinée équatoriale, le Nigéria, le Congo et le Gabon, l'un des pays qui partagent avec le Cameroun une frontière commune. L'allusion au Tchad ne peut que rapprocher le lecteur du Cameroun, terre d'origine de la romancière. À y regarder de près, le cadre spatial rend compte d'un univers plutôt familier, surtout lorsque le lecteur est natif.

Ainsi, des appellations telles que "l'hôpital général de Sombé », «talons de Jésus », « les saos » ou la « Caisse Nationale de Prévoyance Sociale» ne sauraient laisser indifférent le lecteur natif, qui retrouve là des appellations en usage au Cameroun. Ce sont des expressions qui renvoient à des référents précis, se rapportant à l'univers socioculturel camerounais. Les expressions en langue duala du genre de " matanga ma yésu » (traduit par talons de Jésus) et "saos» (qui désignent un fruit d'Afrique) sont la preuve de la volonté, chez la romancière, de marquer l'œuvre de son empreinte culturelle. Derrière l'appellation «Caisse Nationale de Prévoyance Sociale » se cachent des initiales assez bien connues de l'ensemble des Camerounais, la CNPS, autrefois très emblématique, mais dont les immeubles aujourd'hui servent plus au décor qu'à autre chose :

Le commissariat principal de Sombé est un bâtiment jaune, fraîchement repeint pour indiquer que la sécurité est de nouveau une priorité. Il jouxte l'immeuble de la Caisse Nationale de Prévoyance Sociale qui n'a jamais vraiment prévu, et qui n'a rien à verser aux indigents qui sont l'immense majorité des citadins. (CJV, p. 154) 
Les italiques participent de la mise en relief d'une expression empruntée à l'univers social camerounais, de même qu'elles font entendre un discours ironique, que le lecteur natif n'a pas beaucoup de mal à décrypter.

En pointant du doigt la CNPS, l'auteure de Contours $d u$ jour qui vient espère provoquer des réactions d'adhésion, du moins chez le lecteur camerounais, qui connaît assez bien le problème de cette structure. C'est le symbole même de la déroute de toute une nation : l'institution chargée de collecter les cotisations sociales de toutes les entreprises situées au Cameroun et qui devait assurer la protection de tous les salariés et surtout des plus démunis n'est plus que l'ombre d'elle-même. Ce nom évoque, dans l'imaginaire camerounais, la plus grande entreprise d'escroquerie commise envers le peuple. Nombreux sont les Camerounais qui sont morts sans avoir jamais touché le moindre centime de leur droit à la retraite. C'est là un symbole assez fort qui ne peut laisser personne indifférent. Il y a donc chez la romancière le refus d'éviter les sujets tabous : l'Afrique doit oser se regarder en face.

Tout est mis en place dans l'œuvre pour attirer l'attention $\mathrm{du}$ lecteur natif. C'est à croire que le discours onomastique, à travers le choix des noms particulièrement significatifs, porte l'empreinte d'une entreprise de valorisation sociale et culturelle. Ceci n'est pas le fait du hasard, du fait du rôle prépondérant joué par le nom dans les sociétés africaines :

Selon une vieille croyance qui remonte à l'Égypte ancienne, le nom fait partie de la chose nommée : nommer les choses c'est les posséder [...]. La personne ou la chose est censée être à la merci de celui qui en connaît le nom [...]. Car celui qui connaît le nom domine, use de ce pouvoir. Le nom est sacré, c'est pour ne pas le souiller qu'il est tabou. (Bannour, 2005, p. 386) 
À travers l'onomastique, le lecteur natif peut remarquer la manifestation tangible de l'irruption de la culture camerounaise dans le roman. En Afrique, le nom doit signifier quelque chose. On peut comprendre le désarroi de la narratrice face à la situation des peuples à qui on a volé le nom : «Tant de peuples ont disparu, dont les noms ne sont que des inscriptions froides, couchées sur les pages des encyclopédies. » (CJV, p. 53)

S'insurgeant contre ceux qui encouragent les jeunes à déserter le pays, la grand-mère de Musango déclare : «c'est ici que leur nom signifie quelque chose ». La violation du nom serait propre à entraîner l'aliénation culturelle et c'est pour éviter d'en arriver là que Léonora Miano tente de rétablir l'équilibre en utilisant des noms qui ont, pour la plupart, une résonance africaine.

L'héroïne du roman s'emploie au début de l'œuvre à donner l'origine de son nom, de même que sa signification :

Papa racontait autrement mon entrée dans sa vie. Il y avait vu une promesse de bonheur annoncée par le bélier blanc qui avait élu domicile dans notre jardin, pendant plusieurs mois. [...] C'est lui [papa] qui m'a nommée Musango, comme pour signifier son désir de faire taire le tumulte qui l'habitait depuis que l'amour de sa vie l'avait quitté. (CJV, p. 36-37)

La romancière prend le soin d'expliquer au lecteur que Musango signifie « la paix » en langue duala du Cameroun. Ce n'est pas un fait du hasard si elle donne des explications sur certains noms de l'œuvre: en Afrique, un nom n'est jamais le fait du hasard comme le remarquait déjà Miano dans son précédent roman, L'Intérieur de la nuit : «Un nom, d'abord cela devait avoir un sens. Parce que cela avait une incidence sur le caractère, et sur la destinée» (2005, p. 29). L'auteure pousse ainsi le lecteur à aller chercher une signification derrière les 
patronymes. En faisant porter à son héroïne un nom qui signifie la paix, elle adresse un message de paix au lecteur, qu'il soit Camerounais, Français, Africain, Européen ou simplement humain. Il convient de noter en effet que le langage de la paix peut transcender les frontières au-delà des barrières linguistiques et culturelles.

Or, Musango semble bien porter son nom dans le roman. Ce nom va peser sur le destin de l'héroïne, qui se construit d'abord sur la colère et la haine avant de retrouver le chemin de la paix et de l'apaisement. C'est en nommant les événements et les choses qui l'entourent qu'elle réussira à guérir et à s'épanouir :

Nommer la douleur pour pouvoir la chasser, telle est la leçon que tu ne m'as pas enseignée, parce que tu ne l'as pas apprise. Je veux te pardonner, mère, et accepter que ce soit toi la fillette égarée qui n'a jamais grandi. Je veux te pardonner, et remonter avec toi le fleuve houleux de tes peines d'enfant. (CJV, p. 143)

C'est un assez curieux destin que celui de Musango, car, après avoir été chassée de la maison paternelle par sa mère, qui voulait du reste la brûler vive, l'héroïne décide de pardonner à cette mère meurtrière. Par cet acte, elle décide de faire la paix avec elle-même, puis avec sa mère. Musango, c'est donc la paix qui est envoyée dans les familles, dans les continents, dans les pays et, surtout, au milieu des individus. Mais c'est aussi la preuve de l'existence d'un tumulte qu'on souhaite conjurer, comme le souhaitait le père de l'héroïne en faisant le choix de ce nom. Ce terme, emprunté comme beaucoup d'autres à la langue duala, revêt en effet une importance capitale dans la tradition Sawa. 
En effet, les prises de paroles en communauté sont toujours précédées de la formule "musango mu bè na binyo » (la paix soit avec vous!). Ainsi, derrière le nom Musango, semble se trouver inséré un souhait ou, mieux, un vœu de paix. Rien d'étonnant que ce personnage soit le plus accompli dans l'œuvre. C'est le trait d'union entre les différentes générations. Elle vient rétablir les liens rompus par la mère, en créant chez certains le tumulte et, chez d'autres, l'harmonie et la sérénité.

Musango apparaît comme la figure christique, livrée pour expier les fautes des humains. Elle sera acceptée et recueillie par ceux qui poursuivent la vérité et rejetée par ceux qui s'en détournent. Après avoir souffert le martyr comme le Christ, Musango va mourir afin de ressusciter pour le grand bonheur de son peuple. Elle apparaît comme « le Christ grandeur nature qui trônait là, infatigable devant les péchés du monde, inlassablement commis et sans cesse à laver » $(C J V$, p. 84). Cette mort spirituelle est symbolisée dans l'œuvre par le sommeil et par le voyage : «Il me semble que j'ai dormi et voyagé en moimême » (CJV, p. 144), dit l'héroïne après son séjour symbolique dans la grotte. De même que la mort, la transfiguration de Musango rejoint celle du Christ :

Les enfants des pêcheurs me laissent avancer. Je sais qu'ils ne me croient pas réelle. Le soir, ils parleront de moi à leurs parents. Ils me diront grande, blanche, les pieds ne touchant pas la terre. Il leur sera confirmé que je viens des fonds marins, qu'ils ont bien fait de se tenir à distance. (CJV, p. 145)

Miano renouvelle ici l'image de la fée, déjà présente dans l'imaginaire populaire camerounais. En véritable fille des côtes, elle va puiser au plus profond des traditions côtières. L'allusion au «village des pêcheurs", avec ses maisons "montées sur pilotis», au «poisson» et aux «crevettes» ne fait que 
confirmer son appartenance à la culture des côtes camerounaises. Dans l'imaginaire populaire camerounais, la fée habite les fonds marins et son apparition constitue, pour qui sait voir, un signe annonciateur.

Le nom de Musango semble être un don concédé par « Nyambey », le créateur qui a crée les êtres, non pas pour qu'ils soient « de petites choses rampant à la surface du globe » $(\mathrm{CJV}$, p. 12), mais pour qu'ils soient des individus dont l'existence compte. Il faut noter que Nyambey est le terme par lequel le peuple Bassa du Cameroun désigne son Créateur.

D'autres personnages de l'œuvre ont des noms tout aussi évocateurs. C'est par exemple le cas de "Mbalè », dont la romancière indique la signification en langue duala: «la vérité ». En fait, Miano choisit de donner en notes de bas de page la signification de trois noms de personnages, dont Mbalè et Ewenji. On s'en tiendra au choix de l'écrivain en essayant d'en relever la pertinence. La curiosité du lecteur ne peut s'empêcher cependant de comprendre pourquoi avoir choisi d'expliquer uniquement ces trois noms: Musango, Mbalè et Ewenji.

Nous l'avons déjà relevé, " Mbalè » signifie « la vérité ». L'entrée de ce personnage dans le roman se situe à la fin, dans la partie intitulée «Licence». Dans cette partie du roman, l'héroïne présente des signes de maturité et d'aisance qu'elle n'avait pas au début du récit. Deux phrases en relation antonymique permettent de le montrer, «Il n'est que des ombres alentour, c'est à toi que je pense » $(C J V$, p. 15) au début du récit et « L'ombre s'est dissipée alentour, c'est vers toi que je m'élance » $(C J V$, p. 229) à la fin du roman. C'est dans cette période de grande confiance que Musango fait la rencontre de 
Mbalè, de façon assez fortuite, alors qu'elle se dirige vers Embényolo, "la case originelle, la matrice, la source acide» (CJV, p. 231). Sur le chemin, elle est suivie par Mbalè, un adolescent de quinze ans qui la retrouvera plus tard dans la case de la grand-mère. Ce garçon s'est trouvé à plusieurs reprises sur le chemin de l'héroïne : lors de sa fuite au «soul food », dans les rues de Sombé et, maintenant, dans la case de Mbambè. Le lecteur est frappé par la soudaine empathie qui se noue entre les deux adolescents. Musango dira d'ailleurs à ce propos: "C'est la première fois que je m'entends bien avec quelqu'un, que j'ai envie qu'on me connaisse. » (CJV, p. 249)

Pourquoi avoir choisi Mbalè? Probablement, parce que Musango est une adolescente qui aura besoin de faire l'expérience du premier amour. Parce que c'est celui qui veille sur la mère de Musango et qui connaît son secret. Parce que c'est la pierre sur laquelle la grand-mère espère bâtir afin que le «jour qui vient» ne soit pas celui des enfants qui ont envie d'aller «faire l'Europe». Enfin, parce qu'il épousera la signification de son nom pour que les vibrations ne soient pas étouffées, car « [n]ommer un être, c'est le définir, lui indiquer une direction. On est le nom qu'on porte, et il ne faut pas vivre là où ce nom n'est rien, là où sa vibration est étouffée. » $(C J V$, p. 255-256)

Ainsi, Mbalè s'entend comme la vérité avec laquelle le peuple africain doit désormais composer s'il veut exorciser tous ses fantômes: de la traite négrière à la montée des guerres ethniques et à l'émigration clandestine en passant par la lutte pour les indépendances. Car, comme le dit la narratrice, « [n]ous ne disons que la surface des choses qui n'est jamais la vérité » $(C J V$, p. 66). Mbalè, c'est aussi la vérité de l'histoire, à 
laquelle chaque peuple doit avoir accès, la vérité qui seule libère. C'est un nom hautement symbolique, qui ne peut qu'interpeller. Si Musango trouve un certain équilibre à la fin du roman, c'est parce qu'elle a été animée par cette recherche de la vérité : la vérité sur sa naissance, sur le mariage de ses parents, sur sa famille maternelle. Sa grand-mère lui fera remarquer à ce propos: «Une petite assez courageuse pour traverser ce marécage afin de venir à ma rencontre peut affronter la vérité... » $(C J V$, p. 242)

C'est le personnage de Mbalè qui conduit Musango vers l'objet de sa quête: sa mère Ewenji. Accepter la vérité, c'est accepter de se regarder sans se voiler la face. L'envie d'affronter la vérité est ce qui distingue le personnage de Musango de sa mère. Ewenji est décrite dans l'œuvre comme un personnage tourmenté. Ewenji signifie en langue duala «la lutte». Ce personnage semble bien porter son nom, car c'est cette lutte intérieure qui caractérise le personnage en proie à d'énormes souffrances dues à un poids sur la conscience. La femme qui est décrite à travers ce personnage est une mère qui a perdu le sens de la maternité. L'identification peut se faire avec la mère Afrique, qui ne sait pas concerver sa progéniture. La relation entre la mère nourricière et la terre d'Afrique est assez frappante dans ce roman.

Si la lutte caractérise la vie de ce personnage, qui se bat pour avoir un mari, avant de se battre pour être acceptée de sa belle famille, force est de constater que la vie entière de cette femme est une perpétuelle bagarre. Après avoir passé dix mois dans le ventre de sa mère Rachel, elle va par la suite lui imposer deux jours entiers de travail. La maisonnée se composait de douze enfants, que la mère a dû élever toute seule. Enfant, 
Ewenji s'est battue en vain pour se faire une place auprès de sa mère. Les enfants étaient trop nombreux pour pouvoir retenir suffisamment l'attention de leur mère. Devenue femme, Ewenji doit également lutter pour s'accepter et s'assumer en tant que femme. Elle n'y parviendra d'ailleurs pas, ne pouvant assumer ses origines. Elle essaie de rompre les liens avec les siens, qui lui rappellent trop son enfance. C'est une femme qui manque de cran. Cette femme-là ne rappelle que trop l'image d'une Afrique incapable de produire sur son sol des individualités prêtes à prendre en main le destin de ce continent; ce n'est pas pour rien si la narratrice fait constamment référence à l'Afrique pour désigner le Mboasu. Elle parle de l'Afrique comme s'il s'agissait d'une entité agissante :

Le jour se lève et c'est encore la nuit, puisque tu es encore là. Ma mère haineuse, ma mère assassine, ma mère inconsolable d'une souffrance qu'elle ne peut pas nommer. C'est la nuit dans mon esprit où tu prends toutes les formes du chagrin. Je veux marcher vers le fleuve et m'asseoir un moment sur ses berges. Peut-être que j'entendrai ce que disent ces autres enfants mal aimés, ces oubliés dont nul ne porte le deuil. (CJV, p. 125)

Cette citation montre assez bien combien l'analogie entre la mère et l'Afrique est saisissante dans ce roman. Comme la mère, l'Afrique est haineuse (elle ne peut plus retenir ses enfants qui la désertent); comme la mère, l'Afrique est assassine (de nombreux crimes sont perpétrés sur le sol africain au nom de la tradition, de la religion ou de la loi du plus fort); comme la mère, enfin, l'Afrique est inconsolable (elle n'a toujours pas réglé ses comptes avec elle-même et aussi avec l'Occident).

Cette vision globalisante n'a rien de contradictoire. Il ne s'agit nullement de niveler les différentes cultures en présence ni même de confondre les problèmes, mais peut-être de penser 
une harmonisation de toutes ces cultures au service du continent afin de permettre une meilleure communication et une meilleure prise en compte des problèmes africains.

Avec l'onomastique des personnages et des lieux, Léonora Miano donne une impression de vraisemblance en construisant des personnages dont les noms sont porteurs de signification dans le respect des traditions africaines. Elle va jusqu'à imaginer des personnages qui parcourent ses œuvres, comme c'est le cas d'Épa et d'Ayané, qu'une note infrapaginale invite le lecteur à retrouver dans L'Intérieur de la nuit, le précédent roman.

Tout porte à croire que l'auteure invite le lecteur à faire le lien entre les personnages des deux romans et, donc, entre les deux histoires. L'irruption des noms originaires du Cameroun, ajoutée aux expressions empruntées au même univers culturel, permet de constater une sorte de domestication de la langue. À ce titre, on peut constater, avec Odile Cazenave,

un certain glissement, conscient ou non, qui fait ce que les lecteurs considèrent comme une certaine «africanisation » de la langue, qui se produit à partir d'une fabrication en fait hybride, multiple (et qui n'est pas à prendre dans un sens négatif), dont l'objectif est de donner l'impression au lecteur qu'il/ elle découvre la peinture d'une Afrique vraie ou vraisemblable, ici, identifiée spatialement et temporellement comme le Cameroun (2003, p. 154).

Dans cet article, où il était question de cerner un des aspects essentiels de la poétique romanesque de Léonora Miano, nous avons interrogé les artifices mis en place par l'auteure pour rendre compte de son identité et de l'identité collective (de l'Afrique), ce qui nous a permis de mettre en lumière la très nette corrélation existant entre le roman et le milieu social et 
culturel d'appartenance de l'auteure. En effet, le texte de Miano intègre les techniques d'ancrage spatial et actantiel, socioculturel, historique et linguistique. Son art apparaît ainsi comme la manifestation de son enracinement dans son milieu socioculturel, un instrument de transmission de la culture et de l'identité africaines. Il «dégage les valeurs permanentes de l'héritage africain afin que l'élite africaine ne soit pas une élite de déracinés » (E. Mounier, cité par Chevrier, 1987, p.122), mais une élite qui, libérée de tout complexe, arrive à dire la réalité de son continent.

\section{Bibliographie}

Albert, Christiane. (1999), L'Immigration dans le roman francophone contemporain, Paris, Karthala.

Bannour, Abderrazak. (2005), «Tabou et euphémisme ou mise à l'épreuve des limites du possible», dans Sonia Zlitni Fitouri (dir), Le sacré et le profane, Pessac, Presses universitaires de Bordeaux, p. 383-396.

Benveniste, Émile. (1966), Problèmes de linguistique générale 1, Paris, Gallimard.

Beyala, Calixthe. (2003), "L'écriture dans la peau », entretien avec Tirthankar Chanda, Notre Librairie, $\mathrm{n}^{\circ} 151, \mathrm{n} \times 151$, juillet-septembre, p. 35. 
Cazenave, Odile. (2003), Afrique sur Seine: une nouvelle génération de romanciers africains à Paris, Paris, L'Harmattan.

Chevrier, Jacques. (1987), Littérature africaine, Paris, Hatier.

Démoris, René. (2002), Le Roman à la première personne. Du classicisme aux Lumières, Genève, Droz.

Genette, Gérard. (1983), Nouveau discours du récit, Paris, Seuil.

Grawitz, Madeleine. (1990), Méthodes des sciences sociales, Paris, Dalloz.

Guespin, Louis et al. (1971), Dictionnaire de linguistique, Paris, Larousse.

Maingueneau, Dominique. (1991), Pragmatique pour le discours littéraire, Paris, Dunod.

Mendo Zé, Gervais. (2004), «Introduction à la problématique ethnostylistique », Langues et Communication, $\mathrm{n}^{\circ}$ 4, p. 13-26.

Miano, Léonora. (2006), Contours du jour qui vient, Paris, Plon;

—. (2005), L'Intérieur de la nuit, Paris, Plon.

Nabokov, Vladimir. (1985), Littérature I, traduit de l'anglais par Hélène Pasquier, Paris, Fayard.

Ndachi Tagne, David. (1996), Roman et réalités camerounaises, Paris, L'Harmattan.

Noumssi, Gérard Marie. (2004), «Pour une lecture ethnostylistique des Soleils des Indépendances de Ahmadou Kourouna », Langues et communication, vol. $1, \mathrm{n}^{\circ} 4$, p. 81101. 
Peremans, W., E. Van't Dack et W. Clarysse. (1981), Prosopographia Ptolemaica, Tome IX : Addenda et corrigenda au volume III (1956), Leuven, Peeters, coll. "Studia Hellenistica ».

Robert, Marthe. (2004), Roman des origines et origines du roman, Paris, Gallimard.

\title{
Résumé
}

L'article vise à interroger les stylèmes de littérarité propres au texte de Léonora Miano. Il montre que la narration à la première personne et l'onamastique des personnages et des lieux apparaissent comme des procédés fondateurs de l'écriture de cette écrivaine. Ces deux procédés permettent de mettre en lumière la très nette corrélation existant entre le roman et le milieu social et culturel d'appartenance de l'auteure. L'art de Miano apparaît donc comme la manifestation de son enracinement dans son milieu socioculturel, un instrument de transmission de la culture et de l'identité africaines. Il « dégage les valeurs permanentes de l'héritage africain afin que l'élite africaine ne soit pas une élite de déracinés » (E. Mounier, cité par Chevrier, 1987, p. 122), mais une élite qui, libérée de tout complexe, arrive à dire la réalité de son continent.

\begin{abstract}
This paper sets out to study specific literary and stylistic devices in Léonora Miano's novel. In fact, the first-person narrative and the onomastics of characters and places appear to the main devices used by the author in her novel. These two techniques made it possible to bring into light a clear cut relationship between the novel and the socio-cultural
\end{abstract}


environment of the author. Miano's work of art can be viewed as a showcasing of an author who is deep-rooted in her sociocultural setting; a tool for the dissemination of African culture and identity. Her novel «highlights the permanent values of the African heritage so as to prevent the African elite from becoming rootless elite. » (E. Mounier, in Chevrier, 1987, p. 122), but an elite, who, freed of any complex, succeeds in depicting the reality about the continent. 